\title{
INHOMOGENEOUS DISTRIBUTION OF RESIDUAL DEFORMATION EFFECTS IN TEXTURED BCC METALS
}

\author{
Yu. PERLOVICH ${ }^{\mathrm{a}}$, H.J. BUNGE ${ }^{\mathrm{b}, *}$ and M. ISAENKOVA ${ }^{\mathrm{a}}$ \\ a Moscow Engineering Physics Institute, Kashirskoe shosse 31, Moscow \\ 115409, Russia; ${ }^{\mathrm{b}}$ Institut für Metallkunde und Metallphysik, TU Clausthal, \\ Grosser Bruch 23, Clausthal-Zellerfeld 38678, Germany
}

(Received 12 February 1997)

\begin{abstract}
The distribution of residual deformation effects in textured metal sheets was studied by $\mathrm{X}$-ray diffractometric methods using a position-sensitive detector (PSD). The elaborated procedure is based on the geometric principles of texture measurement, while the PSDtechnique allows to observe the profile of the X-ray line simultaneously with registering of its intensity. The proper treatment of experimental results gives values of line intensity, half-width and peak position of each angular position of the studied sample. By analogy with the texture pole figures (TPF), the half-width pole figures (WPF) and the peak position pole figures (PPF) were constructed. The obtained WPFs describe the distribution of deformation effects in the textured sample depending on grain orientation. The distinct correlation between TPF and WPF is demonstrated for rolled Nb and $\beta-\mathrm{Zr}$ : maxima of WPF fall to minima of TPF, while minima of WPF are located at maxima of TPF. The PPF exhibits a cross-wise distribution of elastic strains, providing the mutual equilibrium of extended and contracted regions. To explain this regularity, texture formation mechanisms and probable mutual misorientation of neighbouring grains were considered.
\end{abstract}

Keywords: PSD-technique; Peak broadening pole figures; Peak position pole figures; Niobium; Zirconium alloy; Interrelation between intensity and peak profile parameters

\section{INTRODUCTION}

The inhomogeneous distribution of residual deformation effects in textured metal materials was revealed independently in works by Evstyuchin and Perlovich (1973), Perlovich $(1983 ; 1994)$ and Barral

\footnotetext{
* Corresponding author.
} 
et al. (1986; 1987) using X-ray diffractometric measurements. The principle of the used method consists in registering the X-ray line profile for each position of the studied sample relative to the incident beam in the course of texture measurements. Such a procedure allows to obtain information about lattice distortion for grains with any orientation within the measured region of the pole figure, whereas the X-ray line recorded by the standard method using the focusing geometry after Bragg-Brentano (Taylor, 1961) characterizes only the grains corresponding to the central point of the pole figure. While Perlovich had presented the distributions of the X-ray line half-width in the stereographic projection of rolled molybdenum, Barral et al. constructed the analogous distributions for the mean value of the coherent domain lengths as applied to steel samples, using for their calculation the Warren method. According to Evstyuchin and Perlovich (1973), Perlovich $(1983 ; 1994)$, the comparison of texture pole figures (TPF) with half-width pole figures (WPF) for rolled alloyed molybdenum reveals distinct mutual correlation between integral intensity and halfwidth of X-ray lines: as the orientation of reflecting grains moves from the texture maximum towards the texture minimum, the half-width of the registered X-ray line increases progressively, varying over a very wide range. A similar effect was observed by Barral et al. $(1986 ; 1987)$ : grains of the preferred orientations are less plastically deformed, while grains of the other orientations have higher stored energy. The direct evaluation of substructure inhomogeneity in rolled BCC metals using the X-ray line half-width as a criterium of strain hardening was confirmed repeatedly in the course of some indirect measurements, among which there are studies of inhomogeneous recovery (Perlovich, 1983; 1996) and inhomogeneous martensitic transformations in textured alloys (Liu and Bunge, 1987; Perlovich et al., 1996; Fesenko and Perlovich, 1996).

However, when taking into account that the above-mentioned X-ray measurement of WPF by means of the usual detector is very labourconsuming, it cannot yet be considered as a routine method to study structure inhomogeneities. By the same reason, until recently, only a few investigators tried to obtain such distributions. As a result, the revealed principles of structure inhomogeneity, though being of great importance both for metal science and practice, are not yet very well known. 
Significant progress on this problem was attained however recently by the usage of a position-sensitive detector (PSD) combined with a texture goniometer (Wcislak and Bunge, 1993; 1996). In the present paper the advantages of the PSD-technique for this application will be demonstrated and some original results are discussed.

\section{MEASUREMENT OF LINE BROADENING POLE FIGURES BY POSITION SENSITIVE DETECTOR}

The presented results were obtained by use of the SIEMENS X-ray texture diffactometer D500/TX equipped with a PSD and a multichannel analyser. The simultaneous viewing angle of the PSD is $9^{\circ}$ and the angular resolving power was chosen $0.1^{\circ}$ or $0.05^{\circ}$. This way a diffraction spectrum is obtained for each sample orientation. The subsequent computer treatment of these data provides integral intensity, half-width and peak position, whose distributions are described by texture pole figures (TPF), half-width pole figures (WPF) and peak position pole figures (PPF), respectively. The main stages of the elaborated treatment are characterized below.

\subsection{Smoothing of the Original Experimental Data}

X-ray spectra $I(\theta)$ obtained directly by the PSD show usually significant fluctuations when numbers of pulses registered by neighbouring channels are compared. These fluctuations depend on the size, number and mutual arrangement of grains in reflection position as well as on various instrumental factors. The less is the intensity $I_{n}$ of the X-ray beam diffracted at an angle $2 \theta_{n}$, the higher are relative fluctuations in the vicinity of this angle.

Our measurements include registering of the X-ray line profile for all points of the pole figure, many of which are characterized by a low intensity or/and a significant defocalization effect, so that fluctuations make the further treatment of the obtained data difficult. In order to avoid occasional errors by evaluation of X-ray line parameters, the original spectrum should be "smoothed", i.e. sharp fluctuations should be decreased by means of some mathematical procedure in order to reveal the "true" character of the function $I(\theta)$. In the present work the 
method of spline functions (Remsch, 1967; Anselone and Laurent, 1968) was used for smoothing of the X-ray line profiles measured experimentally.

To describe the experimental data $I(\theta)$, we used a cubic spline function $S(\theta)$ consisting of the partial functions $s_{n}(\theta)$

$$
s_{n}(\theta)=a_{n}\left(\theta-\theta_{n}\right)^{3}+b_{n}\left(\theta-\theta_{n}\right)^{2}+c_{n}\left(\theta-\theta_{n}\right)+d_{n},
$$

each being defined in the interval $\left(\theta_{n}, \theta_{n+1}\right)$, where $\theta_{n}$ and $\theta_{n+1}$ are the experimental points corresponding to neighbouring channels of the PSD and $n=1,2, \ldots, N$. The problem is analyzed in detail in the framework of the mathematical spline theory (Remsch, 1967; Anselone and Laurent, 1968), according to which all parameters $a_{n}, b_{n}, c_{n}$ and $d_{n}$ can be found by solution of the system of matrix equations, which correspond to the condition of the existence of the continuous first and second derivatives of the spline $S(\theta)$ by linking of functions $s_{n}(\theta)$ in all points $\theta_{n}$ as well as to the minimization condition for the following criterion on the whole interval of measurements:

$$
\sum_{n=1}^{N} w_{n}\left\{I_{n}-S\left(\theta_{n}\right)\right\}^{2}+\rho \int_{\theta_{1}}^{\theta_{N}}\left\{S^{\prime \prime}(\theta)\right\}^{2} \mathrm{~d} \theta
$$

where $w_{n}$ is the optional weight for the $n$th measurement and $\rho$ is a smoothing parameter. This criterion consists of two parts: the first characterizes the fit of the curve, and the second the smoothness of the curve. The value of the smoothing parameter $\rho$ weights these two aspects: the larger is $\rho$, the smoother is the final spline curve, though its resulting fit is poorer.

As an example, Fig. 1 exhibits the experimental PSD data $I\left(\theta_{n}\right)$ and splines $S(\theta)$ for the sintered molybdenum tablet used as a standard sample. The presented X-ray line (222) was measured at the sample tilt angle of $50^{\circ}$. The obtained data are shown either as isolated experimental points (a), or as a thin line drawn through these points (b), (c) to be compared with the calculated splines. The displayed splines $S(\theta)$ were calculated with smoothing parameters 0.0003 (b) and 0.01 (c). It is evident that in the considered case the second spline gives a better description of the experimental data than the first one. 


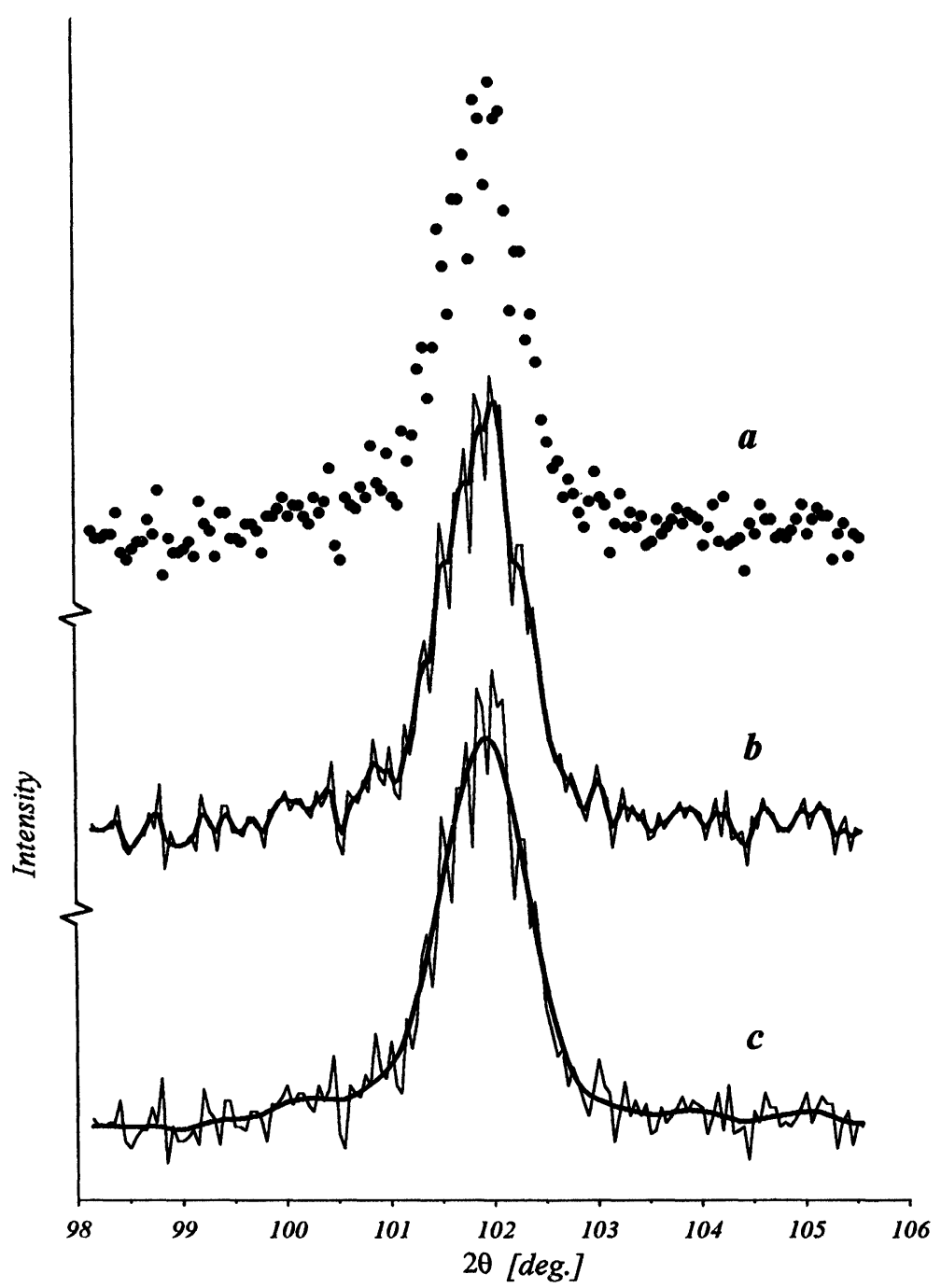

FIGURE 1 Smoothing of experimental data by the spline method with different smoothing parameters: (a) experimental data; (b) $\rho=1 / 30000$; (c) $\rho=1 / 100$.

\subsection{Determination of the Background}

The method to determine the background level has an essential effect on the results of data treatment including doublet separation and 
profile approximation. When X-ray lines to be treated are weak and wide, determination of the background is connected with serious problems. The character of the X-ray lines varies over wide limits depending on the orientation of the reflecting grains and in all cases the used procedure should be efficient as well as be suitable for automatic treatment of large bodies of experimental data. Since in the range of several degrees the background $G(\theta)$ can be assumed to depend linearly on $2 \theta$, the simplest method to determine $G(\theta)$ consists in linear interpolation between points far enough away from any diffraction peak (Wcislak and Bunge, 1993).

Alternatively, in order to draw the background, we used the more formal method by Sonneveld and Visser (1973), which does not require a preliminary analysis of measured spectra in order to find "background points". The method operates only on some fraction of experimental data corrected by the spline; in particular, we used only every 10 th (or 20th) value $S\left(\theta_{n}\right)$, so that the angular distance between them was comparable with the X-ray line half-width. The background values $G_{n}$ were calculated according to the following conditions:

$$
\begin{aligned}
& \text { if }\left(I_{n}+I_{n+20}\right) / 2<I_{n+10} \text {, then } G_{n+10}=\left(I_{n}+I_{n+20}\right) / 2, \\
& \text { if }\left(I_{n}+I_{n+20}\right) / 2>I_{n+10} \text {, then } G_{n+10}=I_{n+10} ; \\
& \quad n=0,10,20 \ldots
\end{aligned}
$$

Repetition of the procedure for $m$ times provides a continuous piecewise smooth curve describing the background. The greater the number of iterations $m$, the lower is the background, approaching in character a straight line. When the measured X-ray line is weak, some instrumental rise of intensity at the central region of the PSD viewing angle becomes noticeable. Such a rise can be taken into account, when calculating the background with a small value $m$ only.

In Fig. 2 the lines of background $G(\theta)$ are drawn as a result of 5 (a) and 15 (b) iterations. In the course of the further treatment, the background-corrected curves $S(\theta)-G(\theta)$ are approximated as a superposition of doublet lines $f_{\alpha 1}$, and $f_{\alpha 2}$. The resulting parameters of the final line $f_{\alpha 1}$ are listed near the curves in Fig. 2. Since the measured $\mathrm{X}$-ray line belongs to the Mo tablet used as a standard sample, its 

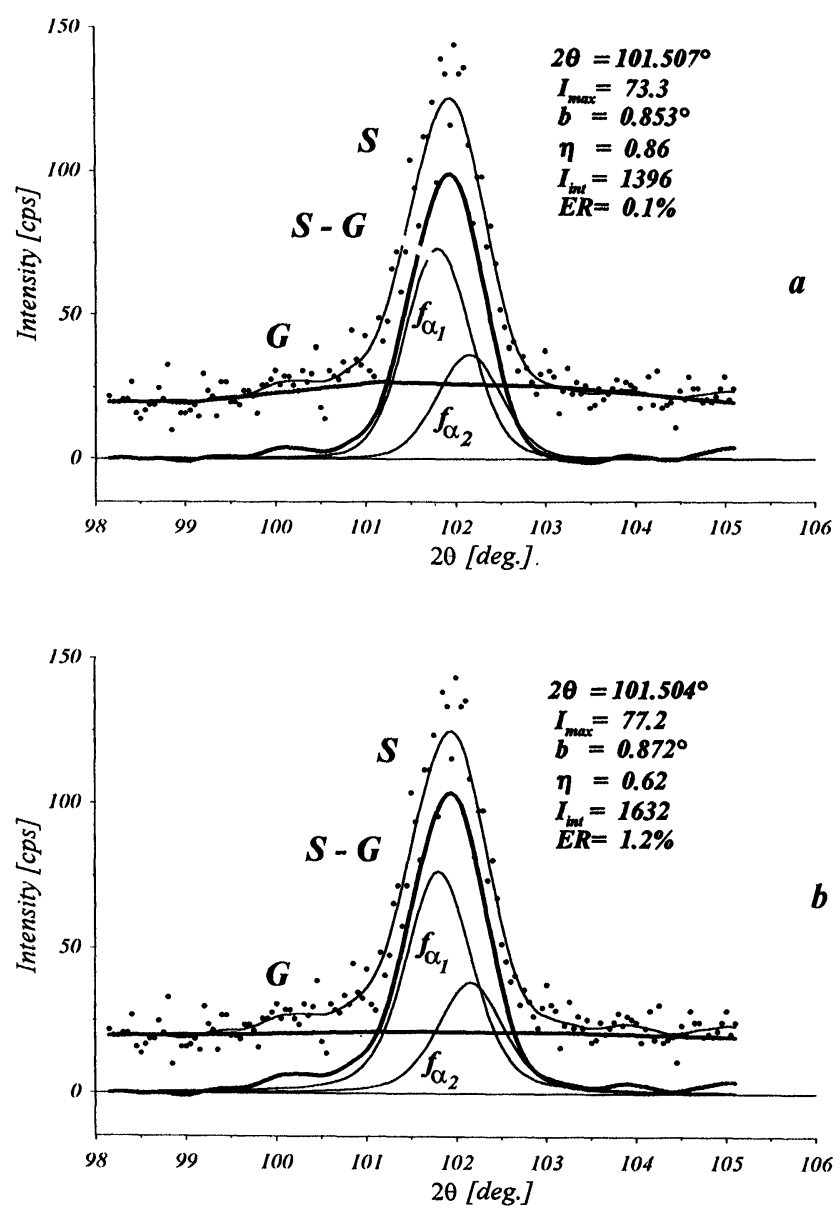

FIGURE 2 The effect of the background position on the parameters of the X-ray profile $f_{\alpha 1}$, found by use of the fitting procedure: (a) $m=5$; (b) $m=15 . S$ - the spline, $\rho=1 / 100 ; G$ - the background; $f_{\alpha 1}$ and $f_{\alpha 2}$ - final doublet lines obtained by approximation of the curve $S-G$.

broadening is assumed to be caused only by instrumental factors and therefore is denoted by the letter $b$ (see Section 2.4). Although the difference between the two background curves appears at first sight to be insignificant, comparison of the respective parameters shows the opposite: lowering of the background results in a sharp rise of the integral intensity $I_{\text {int }}$, as well as a noticeable increase of the half-width $b$. 


\subsection{Approximation of $\mathrm{X}$-ray Lines}

The smoothed diffraction peak profiles were approximated by pseudoVoigt functions:

$$
f_{\mathrm{PV}}(x)=\eta f_{\mathrm{G}}(x)+(1-\eta) f_{\mathrm{C}}(x), \quad x=\theta-\theta_{\max },
$$

where $f_{\mathrm{G}}$ and $f_{\mathrm{C}}$ are Gauss and Cauchy profiles, respectively:

$$
\begin{aligned}
& f_{\mathrm{G}}=\exp \left(-k^{2} x^{2}\right), \\
& f_{\mathrm{C}}=\left(1+k^{2} x^{2}\right)^{-1},
\end{aligned}
$$

and $x$ is the distance from the peak maximum.

The doublet structure of the $K_{\alpha}$-line was taken into account by superposition of two identical pseudo-Voigt profiles $f_{\alpha 1}(x)$ and $f_{\alpha 2}(x-\Delta x)$, the first of which is higher by the factor 2 . Hence, the final model function has the form

$$
f_{\text {mod }}(x)=A f_{\alpha 1}(x)+0.5 A f_{\alpha 1}(x-\Delta x),
$$

where the coefficient $A$ corresponds to the maximal intensity of the $\alpha_{1}$-line and $\Delta x$ is the angular distance between the $\alpha_{1}$ and $\alpha_{2}$ peaks. The model function Eq. (6) contains three free parameters, i.e. $A, \eta$ and $k$, which are determined by the minimum condition

$$
\int\left[f_{\bmod }(x)-f_{\exp }(x)\right]^{2} \mathrm{~d} x=\operatorname{Min},
$$

where $f_{\exp }(x)$ is the experimental (spline-fitted) intensity profile.

Searching of the optimal approximating function $f$ was realized by means of a fitting procedure (Wcislak and Bunge, 1996), involving computer varying of maximal intensity, half-width, peak position and $\eta$-parameter until a minimal fitting error is attained. The fitting error depends on the pole figure angles $\alpha, \beta$ and is described by the fitting error pole figure. In the present case it varied from $2-3 \%$ at texture maxima up to $30 \%$ at texture minima.

\subsection{Determination of the Physical Line Broadening}

The experimentally determined peak profile $f_{\exp }(x)$ (respectively the fitted model function $f_{\text {mod }}(x)$, Eq. (6)) is the convolution of the physical 
line profile $f_{\text {phys }}(x)$ with the instrumental profile function $f_{\text {instr }}(x)$ :

$$
f_{\text {exp }}(x)=\int f_{\text {phys }}(x) f_{\text {instr }}(x-y) \mathrm{d} y .
$$

Correspondingly, the peak width $B$ of the as-measured line $f_{\exp }(x)$ depends on the width $\beta$ of the physical line profile $f_{\text {phys }}(x)$ and on the width $b$ of the instrumental profile $f_{\text {instr }}(x)$. While the first $(\beta)$ is connected with the effects of lattice distortion and coherent domain length (Taylor, 1961), the latter $(b)$ is the resultant of such factors as the divergence of the X-ray beam, the tilt angle, absorption within the specimen and the natural wavelength spread of the used characteristic radiation. In the cases of Gauss and Cauchy functions, respectively, the three half-maximum widths $B, b$ and $\beta$ are related to each other by the simple expressions:

$$
\begin{aligned}
& \text { Gauss } \quad \beta^{2}=B^{2}-b^{2}, \\
& \text { Cauchy } \quad \beta=B-b .
\end{aligned}
$$

When the pseudo-Voigt functions are used for approximation, the physical broadening $\beta$ is calculated usually as the geometric average of values obtained by Eqs. $(9 a, b)$ :

$$
\beta=\left[\left(B^{2}-b^{2}\right)^{1 / 2}(B-b)\right]^{1 / 2} .
$$

If the parameter $\eta$ in Eq. (4) has been determined, alternatively linear interpolation can be used in the form

$$
\beta=\eta\left(B^{2}-b^{2}\right)^{1 / 2}+(1-\eta)(B-b) .
$$

The instrumental broadening $b(\alpha)$, where $\alpha$ is the tilt angle of the sample, can be determined using a standard annealed sample, whose Xray lines are nearly free of physical broadening. The adequate choice of the standard sample is the key point for constructing of correct WPF. When measuring the standard sample, it is necessary to retain a sufficiently high integral intensity of the X-ray line up to the limiting tilt angles as well as to provide a satisfactory precision by determination of the instrumental broadening. 
In the present work the used standard sample was fabricated specially by sintering of a compacted molybdenum powder tablet, while the compacting regime excluded plastic deformation of the particles and the sintering regime restricted the development of grain growth. The tablet combined standards of two types:

(1) a textureless standard which is required usually for TPF correction;

(2) an annealed standard characterized by a negligibly low physical broadening of X-ray lines which is required for WPF correction.

In Fig. 3 correction curves for peak position $\Delta(2 \theta)(\alpha)$ and instrumental broadening $b(\alpha)$ are exhibited, where $\alpha$ is the tilt angle of the sample. The curves were constructed on the basis of data obtained by approximation of three X-ray lines of the standard sample. When the limits of the profile to be treated are chosen properly, the calculated
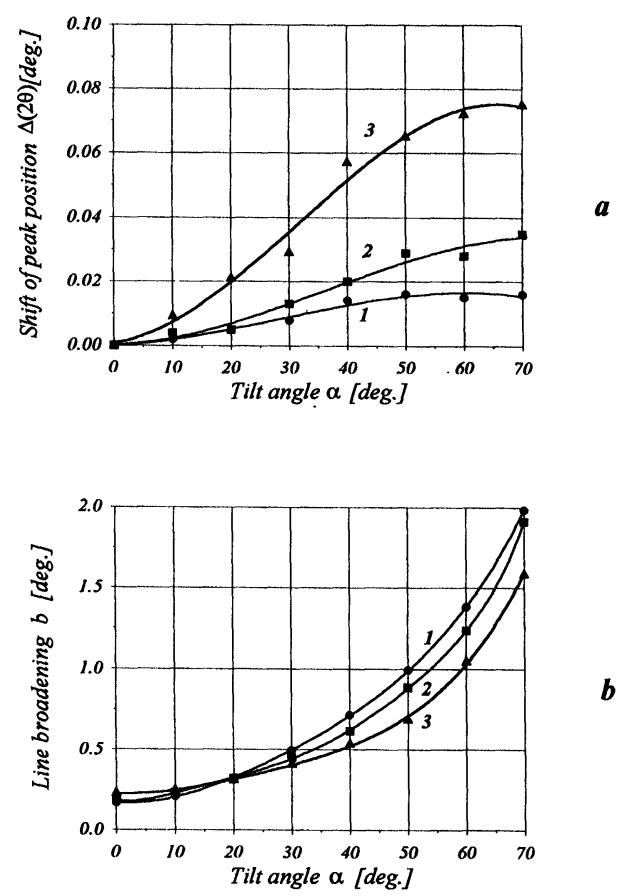

b

FIGURE 3 Correction curves for peak position $\Delta(2 \theta)(\alpha)$ (a) and instrumental broadening $b(\alpha)$; (b) obtained by the treatment of X-ray lines for the Mo standard: (1) $(011), 2 \theta=40.6^{\circ}$; (2) (112), $2 \theta=73.9^{\circ}$; (3) (013), $2 \theta=101.5^{\circ}$. 
integral intensity of lines does not depend on the tilt angle of the sample and, as a result, measured TPFs do not need to be corrected for defocalization.

\subsection{Construction of Pole Figures for X-ray Line Parameters}

The term "pole figure" is used here in a generalized meaning for the distributions of peak profile parameters in the stereographic projection, i.e. half-width pole figure WPF and peak position pole figure PPF, in analogy with the normalized distribution of integral intensity, which is known as texture pole figure (TPF). Since the used diffractometric method allowed to obtain experimental data with an acceptable accuracy only up to tilt angle of $70^{\circ}$, all presented pole figures have an angular radius of $70^{\circ}$, i.e. they are incomplete. Within texture minima the half-width of the X-ray line as well as its peak position sometimes could not be measured because of an insufficient intensity. But in such cases, though the concrete values of the line parameters are not evident from the pole figures, it can be assumed that both the line broadening and the peak position continue their monotonic changes inside the last drawn contour line.

In the present paper our prime interest is with the principal character of the inhomogeneous distribution of residual deformation effects in textured materials as it can be seen in half-width pole figures. The conversion from X-ray line half-widths to concrete substructure characteristics, i.e. values of coherent domain lengths and lattice microdistortions, will not be considered here.

However, the estimates for rolled BCC-metals show that, at least to a first approximation, the following principle is obeyed: the wider is the total physical broadening of the X-ray line, the greater are both its fractions connected with the grain-size effect and with lattice deformation. Therefore, when discussing the distribution of residual microstresses in deformed materials with texture, the peak-width pole figure is taken as a qualitative measure of it.

\section{THE STUDIED MATERIALS}

The above-described approach was applied to investigate the distribution of residual deformation effects in two cold-rolled materials 
with $\mathrm{BCC}$ crystalline lattice $-\mathrm{Nb}$ and the alloy $\mathrm{Zr}-20 \% \mathrm{Nb}$. The $\mathrm{Nb}$ foil, $50 \mu \mathrm{m}$ thick, is used as a substrate for manufacturing $\mathrm{Nb}_{3} \mathrm{Sn}$ superconducting layers, and hence information about its substructure inhomogeneity was important for technological practice. The $\mathrm{Zr}$ $20 \% \mathrm{Nb}$ alloy, having the eutectoid composition, is a convenient model material to study structural features of different phases, which are present in commercial $\mathrm{Zr}$ alloys used as reactor materials. In the quenched condition the alloy consisted of the single high-temperature, $\beta$-phase with BCC lattice, which remained metastable in the course of cold rolling up to high deformation degrees. In order to cause transformation of the metastable $\beta$-phase into the stable $\mathrm{HCP} \alpha$-phase, it is necessary to heat the quenched rolled sample up to $500^{\circ} \mathrm{C}$. The studied samples were cut out of a cold-rolled sheet $100 \mu \mathrm{m}$ thick.

\section{EXPERIMENTAL RESULTS}

The obtained pole figures of integral intensity (TPF), half-width (WPF) and peak position (PPF) for the rolled $\mathrm{Nb}$ foil are shown in Fig. 4(a)(f). The pole figures are constructed by measurements of the X-ray lines (002) and (022). Furthermore, in order to illustrate the correlation between integral intensity and peak broadening, the respective values of Fig. 4(a), (b) and (d), (e) are plotted against each other in Fig. 5(a), (b) (regardless of the position of the points in the pole figures). The analogous pole figures for the quenched rolled $\mathrm{Zr}-20 \% \mathrm{Nb}$ alloy are shown in Fig. 6(a)-(f), while in Fig. 7 the diagrams of correlation between TPF and WPF are plotted. In studies of the $\mathrm{Zr}-20 \% \mathrm{Nb}$ alloy the X-ray lines (002) and (011) were used.

In both materials we deal with the rolling texture of BCC phases, though $\beta-\mathrm{Zr}$, in distinction to $\mathrm{Nb}$, was metastable at the rolling temperature. The main texture components of both materials are $\{001-$ $113-112\}\langle 011\rangle$. Besides that, the rolling texture of the $\mathrm{Nb}$ foil contains the rather strong component $\{011\}\langle 001\rangle$, while the rolling texture of the quenched $\beta-\mathrm{Zr}$ phase contains weak components with the rolling plane $\{111\}$.

The obtained data confirm principally the above-mentioned results by Perlovich et al. and Barral et al., but their statistical significance is 

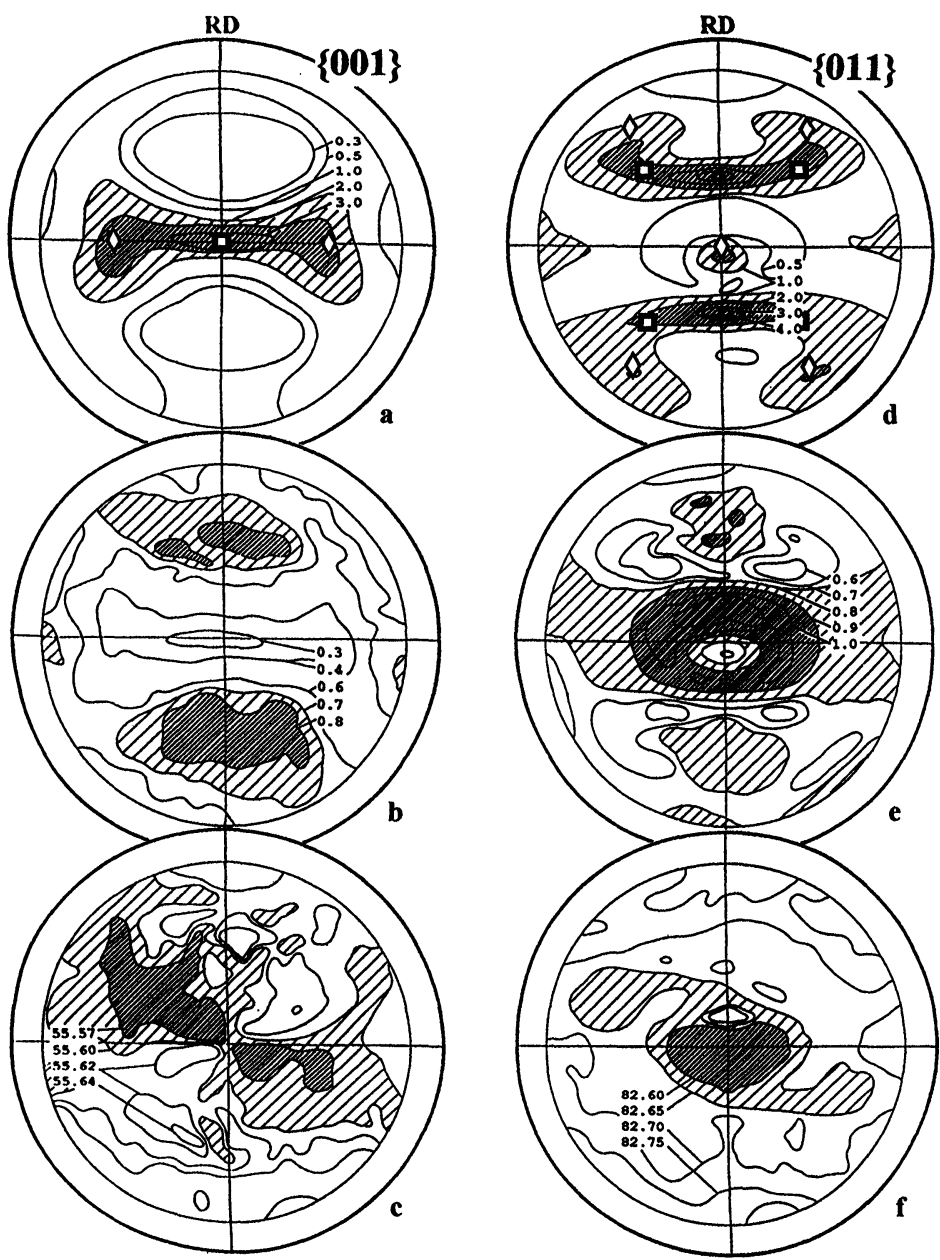

FIGURE 4 Pole figures of the cold-rolled Nb foil: (a) TPF $\{001\}$; (b) WPF (002); (c) PPF (002); (d) TPF \{011\}; (e) WPF (022); (f) PPF (022). $\square-(001)[110]$; $\diamond-$ (011)[100].

essentially higher: repeated PSD-measurements, by the used procedure, were carried out for 1009 points within the incomplete pole figures of each studied sample. Thus, these data cover wide regions of the pole figure, and their reproducibility was checked experimentally. The latter circumstance is of importance, because some contours of TPF and WPF are irregularly shaped and, in order to reveal the character of 

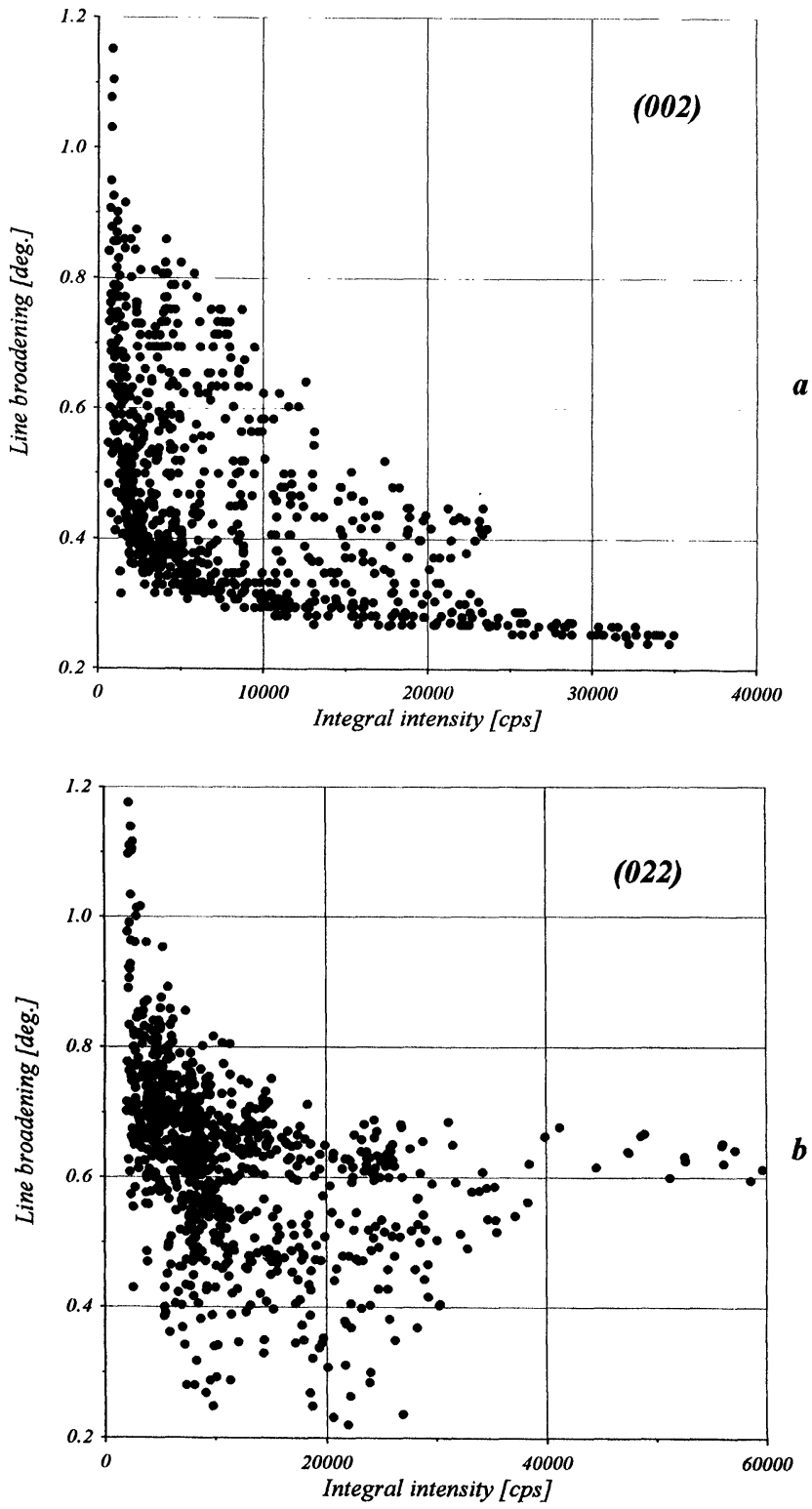

FIGURE 5 Correlation diagrams of physical broadening and integral intensity of $\mathrm{X}$-ray lines for the cold-rolled $\mathrm{Nb}$ foil; the diagrams include 1009 points corresponding to all positions of the sample in the course of texture measurement: (a) (002); (b) (022). 

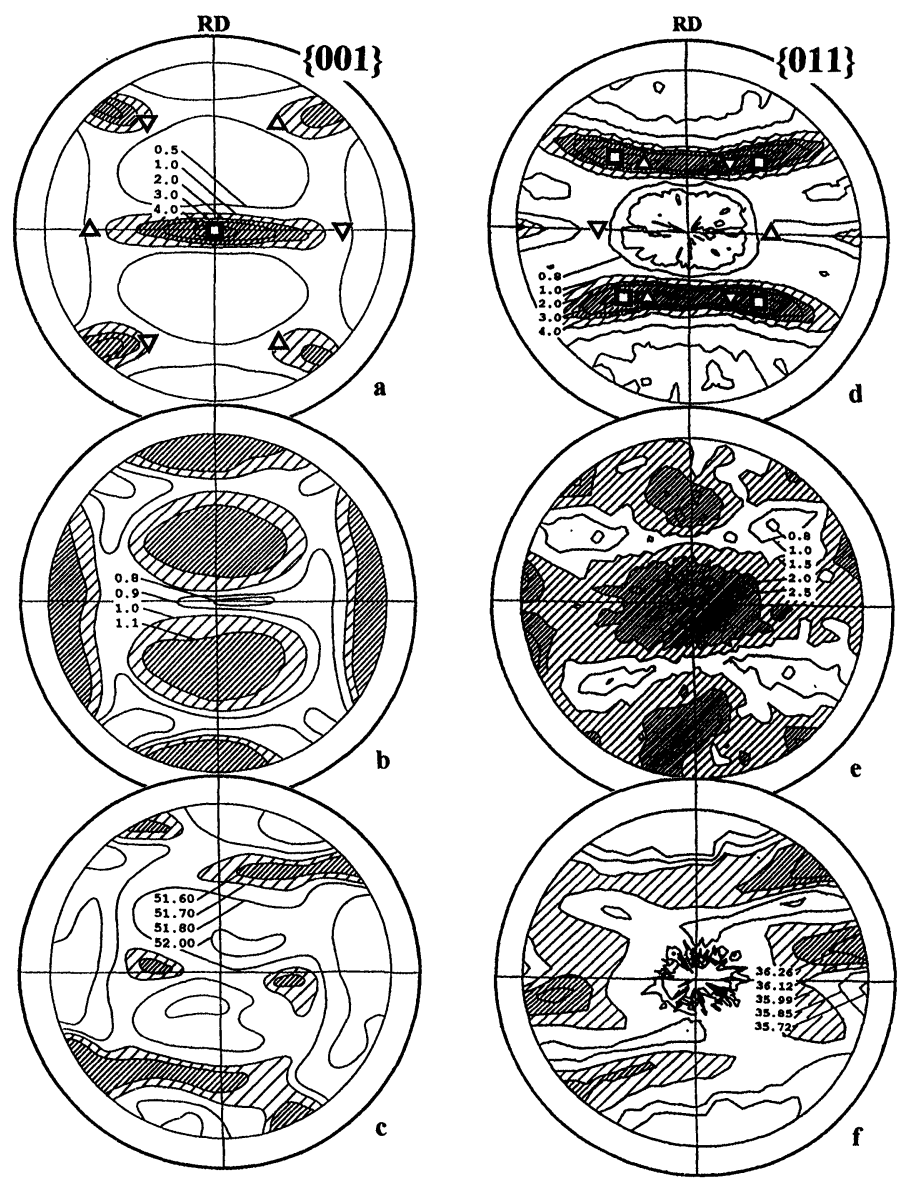

FIGURE 6 Pole figures of the rolled quenched $\mathrm{Zr}-20 \% \mathrm{Nb}$ alloy consisting of $\mathrm{BCC}$ $\beta$-phase: (a) TPF \{001\}; (b) WPF (002); (c) PPF (002); (d) TPF \{011\}; (e) WPF (011); (f) PPF (011). $\square-(001)[110] ; \triangle-(111)[110] ; \nabla-(111)[\overline{1} 10]$.

their mutual correlation, a variety of local distributions should be considered.

The joint consideration of WPFs and corresponding TPFs allows to reveal the following main features of WPFs characterizing the strain hardening distribution in the studied textured materials:

1. The physical broadening of X-ray lines increases as the normal to the reflecting plane moves away from the texture maximum to the neighbouring texture minimum. 
2. Maxima of line broadening are situated within minima of the texture, and conversely, the central regions of texture maxima correspond to the minimal values of line broadening.

3. The physical half-width of X-ray lines varies over the whole range of values accessible with a satisfactory precision, i.e. around $0.2-2^{\circ}$.

4. Contours of equal line broadening in the width pole figures have less regular form than contours of equal pole density, testifying that the concrete values of residual deformation effects correspond only approximately to the regions of texture maxima or minima, respectively.

5. WPFs for different X-ray lines (i.e. (002) and (011) in Fig. 3) correlate to one another, so that the above-stated principle of the inhomogeneous distribution of residual effects refers not only to the orientation of crystallographic axes, but to the grain orientation as well.

6. The diagram in Fig. 7(a) shows two regions with different inclinations testifying that there are two distinct types of the correlation between broadening and integral intensity of the X-ray line. As the integral intensity decreases from high values, the broadening $\beta$ exhibits at first a slow though reliable increase, which occurs to be replaced by a very sharp rise for integral intensities less than $10 \%$ of the maximal value. Hence, line broadening changes in different manners within texture maxima and texture minima indicating different local mechanisms responsible for the dependence of substructure inhomogeneity on grain orientation.

7. This same relationship is also seen in the other correlation diagrams Fig. 5(a),(b) and Fig. 7(b), although in these diagrams the contributions of different texture components are superposed.

The joint consideration of PPFs and TPFs reveals the following characteristics:

1. The most remarkable feature of the obtained peak position pole figures is their specific cross-wise arrangement, consisting in the mutual similarity of first and third as well as second and fourth quadrants.

2. The maxima and minima of the peak position pole figures are found at the slopes of the maxima of the texture pole figures.

3. Opposite slopes of the same texture maximum correspond to opposite peak shifts, i.e. towards higher respectively lower values of the Bragg angle.

These features of peak position pole figures in relation to the texture pole figures were revealed here experimentally for the first time. 

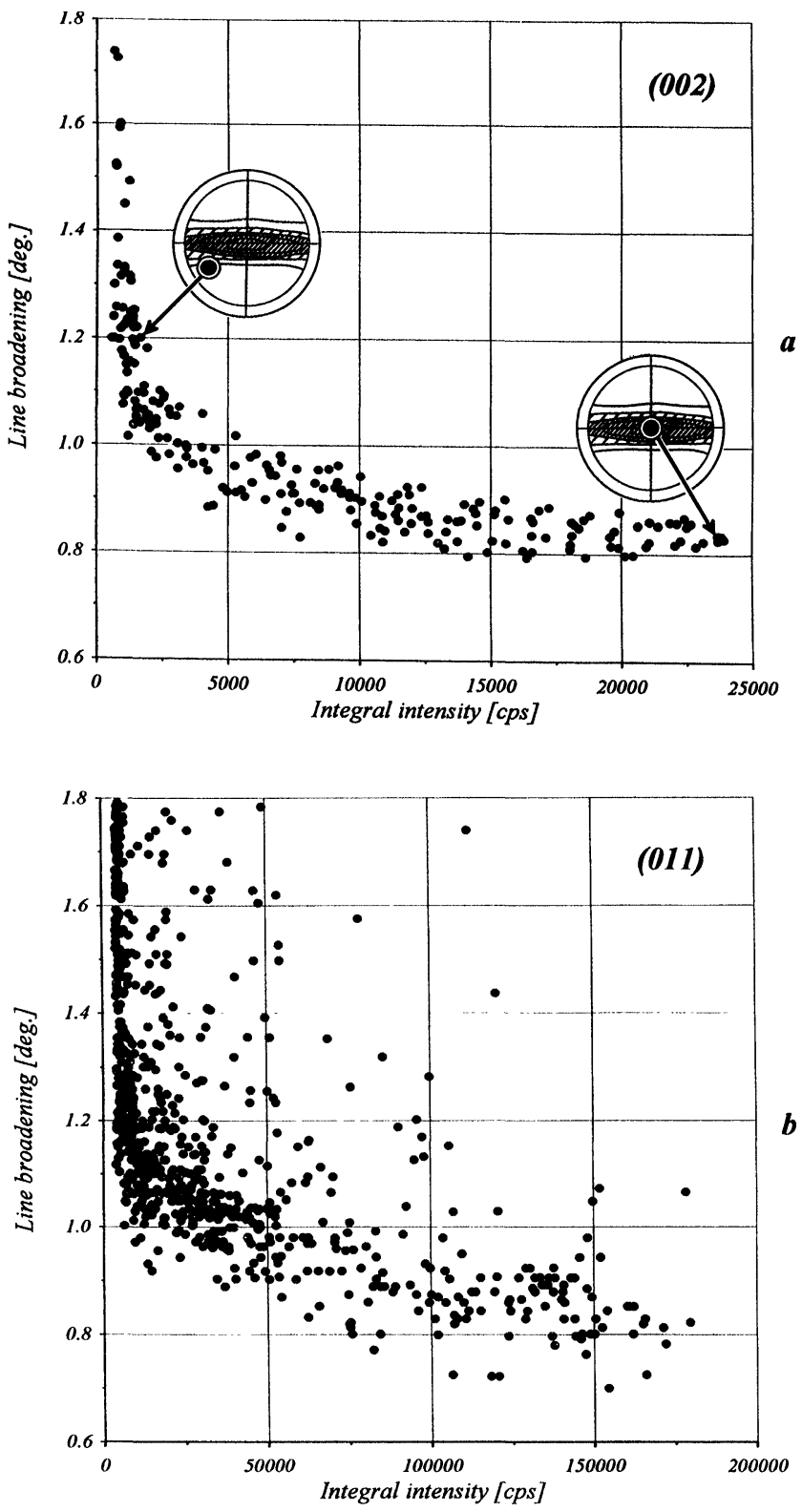

FIGURE 7 Correlation diagrams of physical broadening and integral intensity of $\mathrm{X}$-ray lines for the rolled quenched $\beta$ - Zr phase: (a) (002); (b) (022). Angular radii of treated regions of pole figures: (a) $25^{\circ}$; (b) $60^{\circ}$. 


\section{DISCUSSION OF THE RESULTS}

The presented results require some additional comments concerning the observed features of the obtained pole figures and the assumed character of microstructure, which is responsible for these features.

(1) According to the most frequently used approach (Taylor, 1961), the mean internal stress $\sigma$ is proportional to the fraction of the physical line broadening $\beta_{\mathrm{s}}$, which is caused by the residual lattice distortion $\left(4 \sigma=E \beta_{\mathrm{s}} \operatorname{ctg} \theta\right)$. Since the total physical line broadening, as seen in the width pole figures, can vary by a good order of magnitude, the corresponding variation of residual microstresses, depending on grain orientation, is, at least, the same. Then the local microstresses in the regions corresponding to texture minima can reach the yield point of the material and, in any case, are essentially higher than those estimated by the standard procedure (i.e. considering only the centre of the pole figure). As for the block size $D$, estimated approximately by the relation $D=\lambda / \beta_{\mathrm{D}} \cos \theta$, in the studied rolled materials its variation exceeds surely the range from $0.1 \mu \mathrm{m}$ down to $0.01 \mu \mathrm{m}$, where the line profile is sensitive to the grain-size effect. Thus, the real spectrum of residual deformation effects in rolled metals with texture is extremely wide.

(2) The observed variation of peak position for $\beta-\mathrm{Zr}$ in the limits of $\Delta 2 \theta=0.5^{\circ}$ at $2 \theta_{011}=36^{\circ}$ (Fig. $6(\mathrm{c})$ ) corresponds to a variation of the lattice spacing in the order of $0.5 \%$. Obviously, this value is too high to be assigned entirely to elastic strains. Some contribution to this effect may thus be due to the content of lattice defects by which the volume of the unit cell is changed.

(3) Elastic stresses of 2nd kind, which vary in passing from one (sub)grain to another, should be balanced within a volume of several neighbouring grains relative to some average level determined by elastic stresses of 1st kind (Van Houtte, 1993). Then local deviations of peak position from the average value depending on their signs ("+ " or "-") correspond to additional elastic extension or compression of the grain lattice along considered crystallographic axes. The above-noted crosswise arrangement of the peak position pole figures may be attributed to the necessary equilibrium of tensile and compressive stresses about the planes RD-ND and TD-ND of the sample. As is clear from the obtained pole figures, the elastic stress equilibrium in deformed textured 
polycrystals realizes by means of several modes involving, in particular, division of texture maxima in mutually balanced parts with increased and decreased values of the Bragg angle, the alignment of maxima and minima of PPF in parallel with texture maxima on their slopes, predominance of elastic deformation of one or other sign in successive quadrants of the pole figure, i.e. its cross-wise arrangement.

(4) Conditions of the grain lattice along normals $\langle h k l\rangle$ differ, firstly, depending on grain orientation and, secondly, depending on orientation of the normal within a grain of some component. Thus, half-width pole figures characterize both the substructure inhomogeneity of the deformed polycrystal with a multi-component texture and the substructure anisotropy of grains corresponding to different texturecomponents.

(5) By analysis of the obtained data on the distribution of residual deformation effects, crystallographic normals of the same type $\langle h k l\rangle$ should be distinguished depending on whether they belong to different texture components or to the same one. In the latter case, these normals can differ in distance from the centre of pole figure as well as in their position relative to RD and TD. It seems likely that features of the distribution of X-ray line broadening in the vicinity of the texture maximum depend both on the type of the corresponding texture component and on the position of this maximum in the pole figure. Therefore, in the general case of a multi-component texture some superposition of different effects takes place in WPFs and correlation diagrams, though the same main tendency involving an increase of line broadening in texture minima can be seen always.

(6) The most optimal conditions to study the correlation between integral intensity and peak broadening realize in the vicinity of the isolated texture maximum, as in the central region of TPF $\{001\}_{\beta-z r}$ (Fig. 6(a)). The diagram in Fig. 7(a) is plotted mainly for this region and depicts most correctly the distribution of residual deformation effects depending on grain orientation relative to the texture maximum. An alternative situation takes place in the pole figure $\{001\}_{\mathrm{Nb}}$ (Fig. 4(a)). It contains the more extended central maximum, which accomodates one normal of the component $\{001\}\langle 011\rangle$ coinciding with ND and 2 normals of the component $\{011\}\langle 001\rangle$ located at an angular distance of $45^{\circ}$ from the centre. As a result, the correlation diagram in Fig. 5(a) contains two branches, the lower of which, corresponding to 
the components $\{001-112\}\langle 011\rangle$, is very sharp, whereas the upper one, corresponding to the component $\{011\}\langle 001\rangle$, is scattered. The most complicated character has $\mathrm{WPF}(022)_{\mathrm{Nb}}$ (Fig. 4(e)), because there are three main different positions of normals $\langle 011\rangle$ in $\operatorname{TPF}\{011\}_{\mathrm{Nb}}$ (Fig. 4(d)): 4 normals of the component $\{001\}\langle 011\rangle$ at a distance of $45^{\circ}$ from the centre, one normal of the component $\{011\}\langle 001\rangle$ at the centre and 4 normals of the same component at a distance of $60^{\circ}$ from the centre.

\section{POSSIBLE REASONS FOR THE INHOMOGENEOUS DISTRIBUTION OF RESIDUAL DEFORMATION EFFECTS IN TEXTURED MATERIALS}

The obtained distribution of residual deformation effects can be understood on the basis of a simple model of texture formation. Only some additional modifications of the original scheme of Calnan and Clews $(1950 ; 1951)$ are needed in order to take the inhomogeneity of strain hardening near the texture maxima into account. In the following, two principal mechanisms will be considered, which are assumed to be responsible for the main features of the revealed distributions.

\subsection{The Substructure Inhomogeneity as a Result of the Maintenance of the Final Stable Orientations}

When taking into account that the studied inhomogeneity of residual deformation effects is connected with the texture of the material, it is necessary to take a quick look at the stages of the texture formation process, the main features of which can easily be explained for the case of uniaxial compression.

In case that only a single slip system is acting, the crystal lattice rotates in such a manner, that the normal to the slip plane moves towards the compression axis (Calnan and Clews, 1950). It is the practice to describe the lattice rotation by the trajectory of the loading axis in the space of crystallographic normals, i.e. within the elementary stereographic triangle (for cubic lattices). The Schmid factor for the acting slip system decreases as the lattice rotates and the compression 
axis approaches a boundary of the triangle. Sooner or later the activation of a second slip system becomes possible and the resulting lattice rotation is the geometrical sum of both components. Then the reorientation trajectory goes along the boundary of the stereographic triangle, while the Schmid factor in both active systems continues to decrease. At last the loading axis attains one of the symmetric orientations, corresponding to a minimum in the Schmid factor distribution. Further deformation of the grain then takes place by means of several slip systems. Their concrete number depends on the symmetry of the orientation. The mutual balance of these systems provides the stability of the final orientation, though the minimal Schmid factor plays a leading role in the mechanism of its stability.

Long ago Tucker (1953/1954) had noted that stability of the crystallographic orientation does not mean that the grain keeps the attained orientation constantly, but presumes that the grain returns to this orientation repeatedly, being earlier removed from it owing to disturbance of the temporary equilibrium. This might be a starting position for elaboration of a model, connecting formation of the deformation texture with the development of substructure inhomogeneity (Perlovich, 1983), but a quantitative treatment of the proposed model was not yet realized. Now, that the PSD-technique makes the measurement of WPF a routine procedure, the following model can be used as a basis for the computer simulation of the substructure inhomogeneity development.

From the physical point of view, the maintenance of the stable orientation consists in continuous fluctuations of the grain around this orientation, so that the stable orientation corresponds only to the averaged position of the grain. Various obstacles arise permanently on the way of moving dislocations in the active slip systems, causing their successive retardation and activation. These obstacles result from interactions of dislocations at the microlevel and are statistically distributed. When one of the active slip systems is retarded, the former equilibrium proves to be disturbed and the grain deviates from the stable orientation. Since this orientation is located in a minimum of the Schmid factor distribution, any deviation from it leads inevitably to an increase of the Schmid factor and of the shear stress in the retarded system. As a result, overcoming of the obstacle becomes possible, followed by repeated activation of the system. At the same time, the 
Schmid factor and the shear stresses in other active slip systems decrease in consequence of this deviation, so that some slip retardation takes place in these systems. Thus, the prerequisites for returning of the grain to the initial orientation are fulfilled and, by doing so, to complete the fluctuation cycle.

It should be particularly emphasized that the boundaries of volumes, in which retardation and subsequent reactivation of some slip system occurred, will not coincide precisely. Therefore, every fluctuation of orientation produces new sub-boundaries within the initial grain, contributing to its fragmentation. The greater the number of fluctuations experienced by the grain, the higher are the density of new subboundaries and resulting strain hardening. The angular distance of the crystalline fragment from the ideal symmetric orientation, necessary for repeated activation of the retarded slip system, increases with each successive fluctuation; i.e. the amplitude of fluctuations grows gradually. The attained substructure fragmentation restricts, to a greater or lesser degree, the capacity of the grain to participate in the further deformation and to sustain the stable symmetric orientation. By some critical fragmentation of the initial grain, the newly formed crystallites occur to be too small for further balanced slip. The accessible rise of the Schmid factor is then insufficient for reactivation of the retarded system and, as a result, the crystallite ceases to satisfy the deformation scheme of equally active slip systems and is no longer able to return to the ideal symmetric orientation.

At the next stage this crystallite can deform only by means of asymmetric slip, where the equilibrium takes place betweeen the strengthened slip system having an increased Schmid factor and unstrengthened systems having a decreased Schmid factor owing to the deviation from the symmetric orientation. Though this deviating asymmetric orientation occurs to be stable now, the attainable quantity of slip diminishes since one system is strengthened and the Schmid factor for other systems is decreased. In the deformed textured polycrystal grains with the symmetric orientation correspond to the centre of the texture maximum, while grains with asymmetric orientations correspond to its "slopes". So, the grains corresponding to the central region of the texture maximum make a greater contribution to the plastic deformation of the material, than the grains of the slopes of this maximum. 
According to the preceding, a section through the texture maximum represents a dynamical distribution of crystal orientations. Each of the contributing crystallites has its particular orientation at the instant of observation only occasionally. At the next moment the crystallite can pass to some neighbouring part of the texture maximum, while other crystallite would occupy its former place in the space of orientations. At the same time, if the final deformation texture has formed already, the general configuration of the texture maximum, being a statistical distribution, changes only rather slowly. Nevertheless, some additional scattering of the texture maximum should take place because of the gradual strain hardening and further fragmentation. As the grain fragmentation continues, the smallest newborn subgrains, which can no longer take an active part in the further plastic deformation, begin to change their orientation in an occasional manner. The texture minima are filled namely by those subgrains, which do not conform to the crystallographic regularities of reorientation.

Thus, the observed inhomogeneous grain fragmentation, depending on their position relative to texture maxima and minima, seems to be a quite natural phenomenon accompanying the deformation texture development. It can be predicted by taking into account the Schmid factor distribution for acting slip systems.

\subsection{Inhomogeneous Distribution of Residual Stresses Owing to Deformation Incompatibility of Neighbouring Grains}

The distribution of residual stresses in deformed polycrystals depends on the mutual misorientation of neighbouring grains. This misorientation, in turn, depends on the totality of microstructural parameters including subgrain shape, contents of low-angle and high-angle boundaries as well as the texture. These parameters are interconnected to a large degree, so that, for example, some texture features are determined by the character of present grain boundaries. In the course of deformation, slip contributes mainly to the formation of low-angle boundaries, while twinning creates new high-angle boundaries in addition to those existing initially.

When taking into account that the rolled metal sheet with the developed deformation texture has, as a rule, a layered microstructure consisting of plate-like grains parallel to the rolling plane, it can be 
assumed, as an approximation, that the misorientation of neighbouring grains reflects chiefly the relative intensity of different texture components. The probability to find a subgrain with an orientation within the texture maximum is many times higher than the probability to meet a subgrain from the texture minimum. Consequently, in the case of a single-component texture a subgrain belonging to the texture maximum is bordered predominantly by subgrains of the same component. As a result, their mutual misorientation occurs to be comparatively low and does not exceed the angular width of this maximum. At the same time, the most probable neighbours of subgrain belonging to the texture minimum are the subgrains from the texture maximum. Therefore, the misorientation at the boundaries of this subgrain is close to an angular distance between the texture minimum and maximum, i.e. in the average it attains the values corresponding to highangle boundaries.

(Sub)grains with different orientations, in particular belonging to texture maxima and minima, respond to any external loading in different manners owing both to the plastic anisotropy and to their aboveconsidered substructure differences. The deformation incompatibility of neighbouring grains results in the rise of internal intergranular stresses. But numerous coarse subgrains of the texture maximum "do not notice" rare small inclusions or thin interlayers with orientations corresponding to the texture minimum. And conversely, the subgrains from the texture minimum exhibit significant internal microstresses initiated by the interaction with surrounding subgrains having orientations within the texture maximum.

The foregoing explains the principles, which can be used as a basis for quantitative simulation of the development of substructure inhomogeneity by plastic deformation. But, while the principles of modelling as applied to inhomogeneous grain fragmentation seem to be quite clear already, the simulation procedure for the distribution of residual stresses is more complicated.

\section{SUMMARY}

1. The application of X-ray diffractometry with PSD-technique proves to be the most advantageous method to study systematically the 
inhomogeneous distribution of residual deformation effects in textured metals depending on grain orientation, provided that the experimental data are carefully corrected for instrumental factors.

2. The presented pole figures of X-ray line physical broadening for cold-rolled $\mathrm{Nb}$ and $\beta$ - $\mathrm{Zr}$ show detailed correlation with the usual texture pole figure, testifying that the distribution of residual deformation effects develops in connection with the rolling texture formation.

3. The main feature of the revealed distribution consists in the amplification of residual effects as the grain orientation moves away from the texture maximum towards the texture minimum.

4. The peak position pole figure exhibits the mutual equilibrium of extended and contracted regions about the rolling and transverse directions, having regard, as well, to the character of the texture maxima.

5. A model is proposed to explain the principal character of the observed substructure inhomogeneity by the action of mechanisms responsible for the maintenance of final stable orientations.

6. The inhomogeneous distribution of residual microstresses in textured material is conditioned by the deformation incompatibility of neighbouring crystallites with different orientations.

\section{Acknowledgements}

The authors gratefully acknowledge financial support of the project by the German Research Foundation, DFG. Also they are grateful to T. Leffers of Risoe National Laboratory (Denmark) for stimulating discussions about the mechanisms responsible for the inhomogeneous distribution of residual stresses in textured materials.

\section{References}

Anselone, P.M. and Laurent, P.J. (1968). A general method for the construction of interpolating or smoothing spline functions. Num. Math., 12, 66-82.

Barral, M., Sprauel, J.M., Lebrun, J.L. and Maeder, G. (1986). X-ray macrostress determination and microstrain evaluation on a textured material. In: Experimental Techniques of Texture Analysis. Ed., H.J. Bunge, Deutsche Gesellshaft für Metallkunde, Informationsgesellschaft Verlag, pp. 419-428.

Barral, M., Lebrun, J.L., Sprauel, J.M. and Maeder, G. (1987). X-ray stress analysis of textured materials. Calculations of X-ray compliances with the ODF. In: Theoretical Methods of Texture Analysis. Ed., H.J. Bunge, Deutsche Gesellshaft für Metallkunde, Informationgesellschaft Verlag, pp. 355-364. 
Calnan, E.A. and Clews, C.J.B. (1950). Deformation textures in face-centred cubic metals. Phil. Mag. Ser., 7, 41, 322, 1085-1100.

Calnan, E.A. and Clews, C.J.B. (1951). The development of deformation textures in metals. Part II. Body-centred cubic metals. Phil. Mag. Ser., 7, 42, 329, 616-635.

Evstyuchin, A.I. and Perlovich, Yu. (1973). X-ray method for the selective investigation of grains with given crystallographic orientations as applied to rolled materials. In: Metallurgy and Metal Science of Pure Metals. Atomizdat, Moscow, pp. 32-38.

Fesenko, V. and Perlovich, Yu. (1996). Martensitic transformation development in the rolled $\mathrm{Ti}-\mathrm{Ni}$ alloy: Varying of temperature parameters depending on grain orientation. JOURNAL DE PHYSIQUE IV, Colloque C1, supplement au Journal de Physique III, 6, 197-202.

Liu, W.P. and Bunge, H.J. (1987). Orientation selective martensite transformation. The transformation probability function. In: Theoretical Methods of Texture Analysis. Ed., H.J. Bunge, Deutsche Gesellschaft für Metallkunde, Informationsgesellschaft Verlag, pp. 337-351.

Perlovich, Yu. (1983). Inhomogeneity of strain hardening, recovery and recrystallization in alloyed molybdenum with the developed crystallographic texture. In: Structure, Texture and Mechanical Properties of Deformed Molybdenum Alloys. Naukova Dumka, Kiev, pp. 87-145.

Perlovich, Yu. (1994). Development of strain hardening inhomogeneity during texture formation under rolling of BCC-metals. In: Numerical Prediction of Deformation Processes and the Behaviour of Real Materials. Proceedings of the 15th Riso International Symposium on Materials Science. Eds., S.I. Andersen et al., Riso National Laboratory, Roskilde, Denmark, pp. 445-450.

Perlovich, Yu., Bunge, H.J., Isaenkova, M., Park, N.J., Wcislak, L. and Zuev, M. (1996). Inhomogeneity of phase transformations $\beta \rightarrow \omega$ and $\beta \rightarrow \alpha$ in the quenched coldrolled alloy $\mathrm{Zr}-20 \% \mathrm{Nb}$. JOURNAL DE PHYSIQUE IV, Colloque C1, supplement au Journal de Physique III, 6, 149-156.

Perlovich, Yu. (1996). Some physical errors of X-ray texture measurements. Textures and Microstructures, 25, 129-147.

Remsch, C.H. (1967). Smoothing by spline functions. Num. Math., 10, 177-183.

Sonneveld, E.J. and Visser, J.W. (1973). Automatic collection of powder data from photographs. J. Appl. Cryst., 8, 1-7.

Taylor, A. (1961). X-ray Metallography. John Wiley \& Sons, Inc., New York, London, $993 \mathrm{p}$.

Tucker, J.E. (1953/1954). Discussion on preferred orientation. J. Inst. Metals, 82, pt.13, $655-656$

Van Houtte, P. (1993). Stress measurements in textured materials. Materials Science Forum, 133-136, 97-110.

Wcislak, L., Bunge, H.J. and Nauer-Gerhardt, C. (1993). X-ray diffraction texture analysis with a position sensitive detector. Z. Metallkunde, 84, 479-493.

Wcislak, L. and Bunge, H.J. (1996). Texture Analysis with a Position Sensitive Detector. Cuvillier Verlag, Göttingen. 